\title{
ORIGINAL ARTICLE \\ Computed tomographic angiography of anterior spinal artery in acute cervical spinal cord injury
}

\author{
Z Zhang, H Wang, Y Zhou and J Wang
}

Background: In addition to spinal fracture and/or dislocation, acute traumatic spinal cord injury (SCI) includes neural tissue and vascular damage at the site of contusion. Vascular damage results in spinal cord ischemia, which is a key factor that contributes to secondary pathogenesis. There is a need to identify spinal cord ischemia secondary to anterior spinal artery (ASA) rupture in acute cervical SCl.

Methods: After admission, 20 patients with cervical SCI, including 10 cases of central cord syndrome (CCS), four cases of brown-sequard syndrome (BSS) and six cases of tetraplegia (ASIA A), had computed tomographic angiography (CTA) of ASA performed on them. ASA rupture or occlusion was observed.

Results: The ASA was visualized in all 20 patients. No ASA rupture was found in CCS and BSS patients, even for sever blunt cervical fracture and dislocation tetraplegia patients, except one stab-wound patient.

Conclusion: CTA provides the most detailed, highest resolution imaging of ASA in acute cervical SCI. ASA rupture is not commonly seen in acute blunt cervical SCl.

Spinal Cord (2013) 51, 442-447; doi:10.1038/sc.2012.179; published online 22 January 2013

Keywords: Spinal cord injury; CT angiography; anterior spinal artery; cervical spine

\section{INTRODUCTION}

Acute traumatic spinal cord injury (SCI), includes primary and secondary injuries. The primary mechanical injury results in axonal and vascular damage at the site of contusion and is generally considered to be irreversible. In the minutes, weeks or even years following the initial injury, the secondary injury can include a cascade of a series of pathobiological events, such as ischemia, vasospasm, delayed axonal loss, apoptosis, ion-mediated cell damage, excitotoxicity, neuroinflammation, mitochondrial dysfunction and oxidative cell damage. ${ }^{1}$ Among these pathobiological events, ischemia is a key factor and key contributor to the secondary pathogenesis, which influences the chronic functional deficits in individuals who have experienced SCI. The anterior spinal arteries (ASA) supply the anterior horn and the anterior part of the lateral column on the left or right side at each level of the spinal cord. However, whether spinal cord ischemia is secondary to ASA rupture in SCI remains unknown. There are a number of papers that, for detecting spinal cord infarction of acute spinal cord ischemia syndrome, relied upon autopsy, catheter angiography and magnetic resonance imaging (MRI) of ASA.,3 Compared with MR angiography (MRA), computed tomographic angiography (CTA) has higher spatial resolution and is capable of covisualizing the anatomy (that is, spinal cord and vertebral bone) in addition to the spinal vessels. MRA is beneficial for the visualization of submillimeter-to-millimeter-sized intradural vessels and helpful for indicating the location of the vascular lesion. ${ }^{4}$ We hypothesize that spinal cord ischemia may result in ASA rupture in acute traumatic SCI. We also hypothesize that CTA of ASA is the ideal method of covisualizing both ASA and injured bones. This study aims to identify ASA rupture by CTA in acute cervical SCI.

\section{MATERIALS AND METHODS}

\section{Patients}

Twenty patients with cervical SCI were admitted to our hospital between 2008 and 2009. The clinical characteristics of the patients are summarized in Table 1. In these 20 patients( 18 males and 2 females), the distribution of cervical trauma was from $\mathrm{C} 2$ to $\mathrm{C} 7$; the duration from injury until surgery ranged from 5 days to 6 months; follow-up ranged from 8-13 months (mean, 10 months) All patients underwent plain cervical radiographs, CT scans and 1.5-Tesla MRI. T1-weighted and T2-weighted MRIs were performed to define spinal cord compression and the extent of intramedullary signal intensity change. The mode of acute traumatic SCI was defined as central cord syndrome (CCS) (10 cases), brown-sequard syndrome (BSS) (4 cases) and tetraplegia (ASIA A) (6 cases). All patients arriving within $8 \mathrm{~h}$ of trauma received high-dose methylprednisolone. Anterior decompression and fusion or/and posterior screw fixation surgery was performed on the majority of patients. The timing of surgery depended on the severity of the neurological injury. Preoperative and postoperative neurological status was evaluated using the American Spinal Injury Association (ASIA) impairment classification and the motor score. ${ }^{5}$

\section{CTA of ASA}

With the institutional review board approval of the research protocol, CTA of ASA was performed on the patients. One or two days after patients underwent MRI, patients were scanned with 64-slice MDCT scanners (LightSpeed 64, GE Medical System, Milwaukee, WI, USA). All CT scans were obtained with $0.5 \mathrm{~s}$ rotation, $0.625 \mathrm{~mm}$ nominal detector widths, pitch of $0.984,120 \mathrm{kV}$ and $480 \mathrm{~mA}$. Transverse sections were reconstructed with $50 \%$ overlap relative to the effective section thickness of $0.6 \mathrm{~mm}$. Iohexol (Amersham Health, 


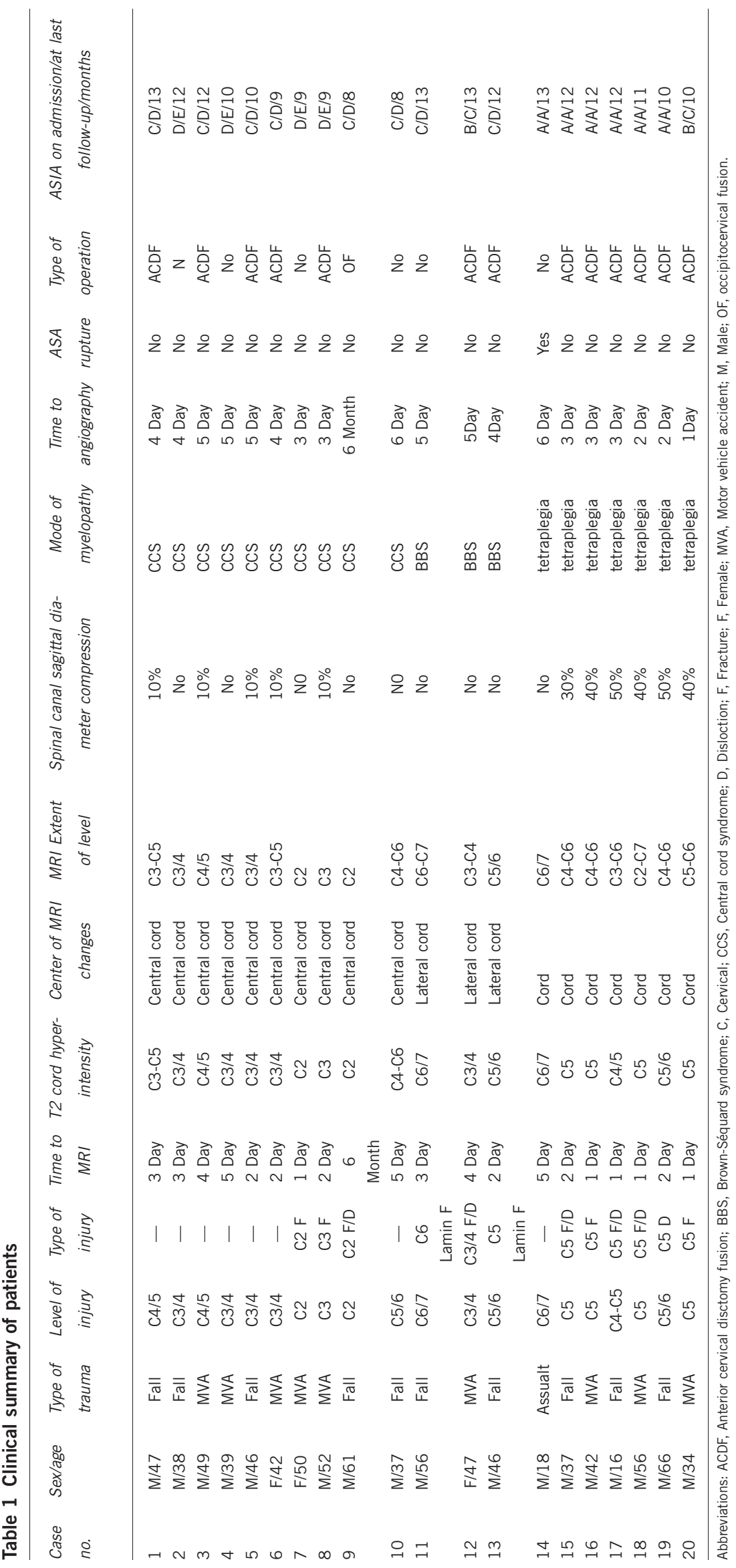




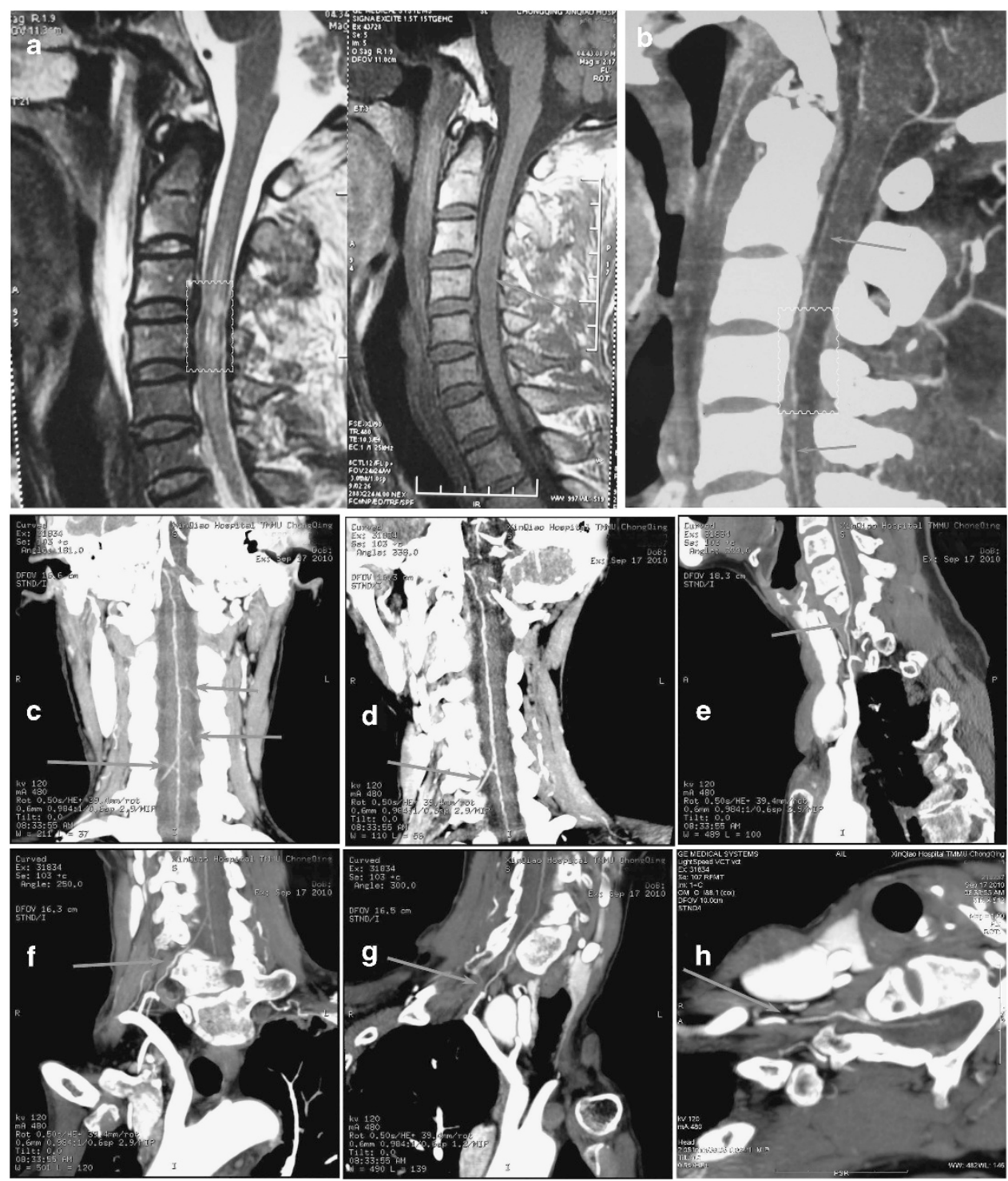

Figure 1 Patient 1. A 47-year-old man with CCS at C3-C5.(a) Sagittal T2-weighted MRI showing hyperintensity in the central cord area at C3-C5 (square frame), while T1-weighted MRI show atypical intramedullary hypointensity (arrow). (b) Sagittal view of CTA showing no ASA rupture (arrows). (c-h) Coronal, obliquesagittal and axial views of CTA showing adamkiewicz artery (arrows) as the ASA suppliers. A full color version of this figure is available at the Spinal Cord journal online.

Princeton, NJ, USA) was administered through an antecubital vein with a dose of $120-150 \mathrm{ml}\left(350 \mathrm{mgI} \mathrm{ml}^{-1}\right)$ at a rate of $5 \mathrm{ml} \mathrm{s}^{-1}$. The scan delay was determined by a preliminary $20 \mathrm{ml}$ test injection at the level of basilar artery. All observations were made retrospectively by two neuroradiologists. To examine the ASA transverse sections, multiplanar reformations, curved planar reformation and thin-slab $(2-4 \mathrm{~mm})$ maximum intensity projections were generated and displayed on a workstation (Advantage Workstation 4.3, GE Medical Systems) with window and level settings selected to maximize arterial to background discrimination. The ASA was identified by maintaining the maximum intensity projection-slab parallel to the anterior surface of the spinal cord at each vertebral level assessed from $\mathrm{C} 1$ to $\mathrm{C} 7$. An enhanced artery on the midline ventral surface of the spinal cord was interpreted as the ASA. At the same time, an artery originating from the aorta and coursing through the intervertebral foramen to join the ASA in a hairpin configuration was interpreted as the adamkiewicz artery (Figures 1d, e, f). Covisualizations of ASA and adamkiewicz artery were used to identify ASA; covisualizations of veins were not criteria of identifying ASA. Another criterion was that the ASA originated from vertebral artery in skull base. Inter-rater reliability, in the form of consensual identification of ASA by two radiologists, was required.

\section{Statistical analysis}

Comparisons were performed by using Student's $t$ tests and linear regression analyses. We reported the results by using $P<0.05$ as the criterion for a significant difference. The odds ratio was reported with $95 \%$ confidence intervals.

\section{RESULTS}

\section{ASA visualization}

The clinical data are summarized in Table 1. The ASA was visualized in all 20 patients. The course of the ASA extended from $\mathrm{C} 1$ to $\mathrm{C} 7$ in the sagittal, coronal and transverse views. The ASA runs along the entire length of the anterior surface of the 

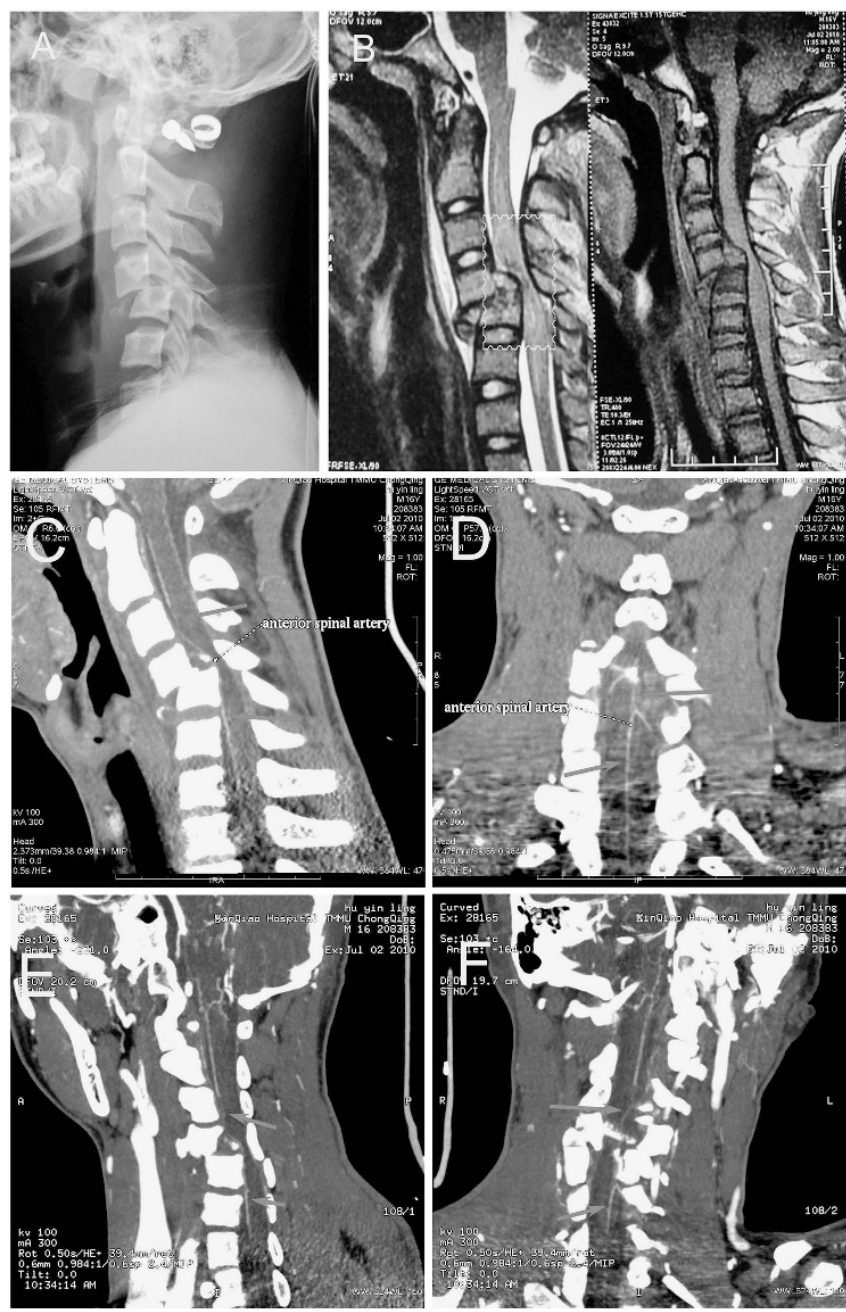

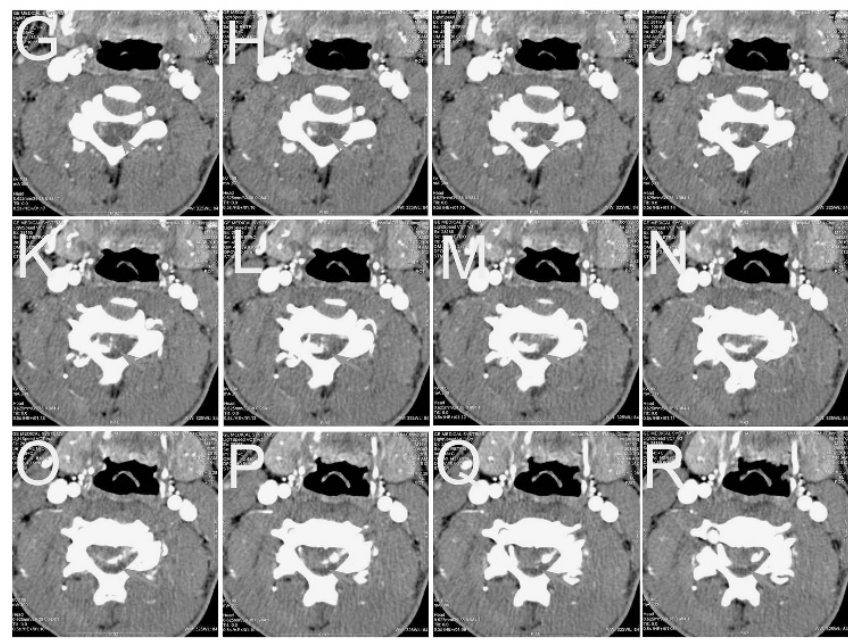

Figure 2 Patient 17. A 16-year-old man with tetraplegia at C4 fracture and dislocation. (A) Lateral radiograph showing C5 fracture and dislocation. (B) Sagittal T1 and T2-weighted MRI showing cord hyperintensity at C3-C6 (square frame). (C-F) Sagittal, coronal and oblique views of CTA showing no ASA rupture (arrows). (G-R) Axial views of CTA in region of C4 to C5 by order showing no ASA rupture (arrows). A full color version of this figure is available at the Spinal Cord journal online.

spinal cord. Anterior radiculomedullary arteries (ARAs), which supply the spinal cord throughout its length by anastomoses branching upward and downward, were not visualized for the angiography, because very little or even no blood flows from ARAs in either direction. However, arteries with iohexol in the transverse view were found, which may be part of ARAs. No ASA rupture was found in CCS (Figure 1) and BSS, even for sever cervical fracture and dislocation patients with spinal cord compression (Figure 2), except one stab-wound patient (Figure 3). Adamkiewicz artery was also visualized in most cases; no ruptures were found in any patients.

\section{Correlation between spinal cord ischemia and ASA rupture}

SCI includes edema, myelomalacia, gliosis or inflammation, which present as irregular T2 hyperintensity in MRI and in the low signal intensity area in T1-weighted images. These intramedullary signal intensity changes can also be seen as MRI-based predictors of spinal cord ischemia. ${ }^{6}$ Seeing as ARAs were not visualized and no ASA ruptures were found in any BSS, CCS and tetraplegia patients (except one stab-wound patient), our evidence shows that spinal cord ischemia is not associated with ASA rupture(Figures 1-3). Predictors of SCI that can be determined through MRI (such as location and extent of the cord edema) are correlated with primary SCI and, perhaps, the reduction of ARAs blood supply. ARAs maybe ruptured, occluded or compressed by swelling of the surrounding tissue.

\section{Neurological prognosis}

There is a close correlation between spinal cord ischemia that can be determined through MRI and neurological prognosis. Subtle spinal cord ischemia BBS and CCS patients usually had less severe neurological injuries and displayed faster improvement, while distinct spinal cord ischemia tetraplegia patients had severe neurological injuries and displayed very slow or no improvement (Table 1$)(P<0.05)$. No correlation was found between ASA and neurological prognosis, except in one stab-wound patient $(P>0.05)$.

\section{DISCUSSION}

\section{CTA of ASA}

The ASA (diameter, $0.2-0.8 \mathrm{~mm}$ ) runs along the entire length of the anterior surface of the spinal cord and distributes blood to the 

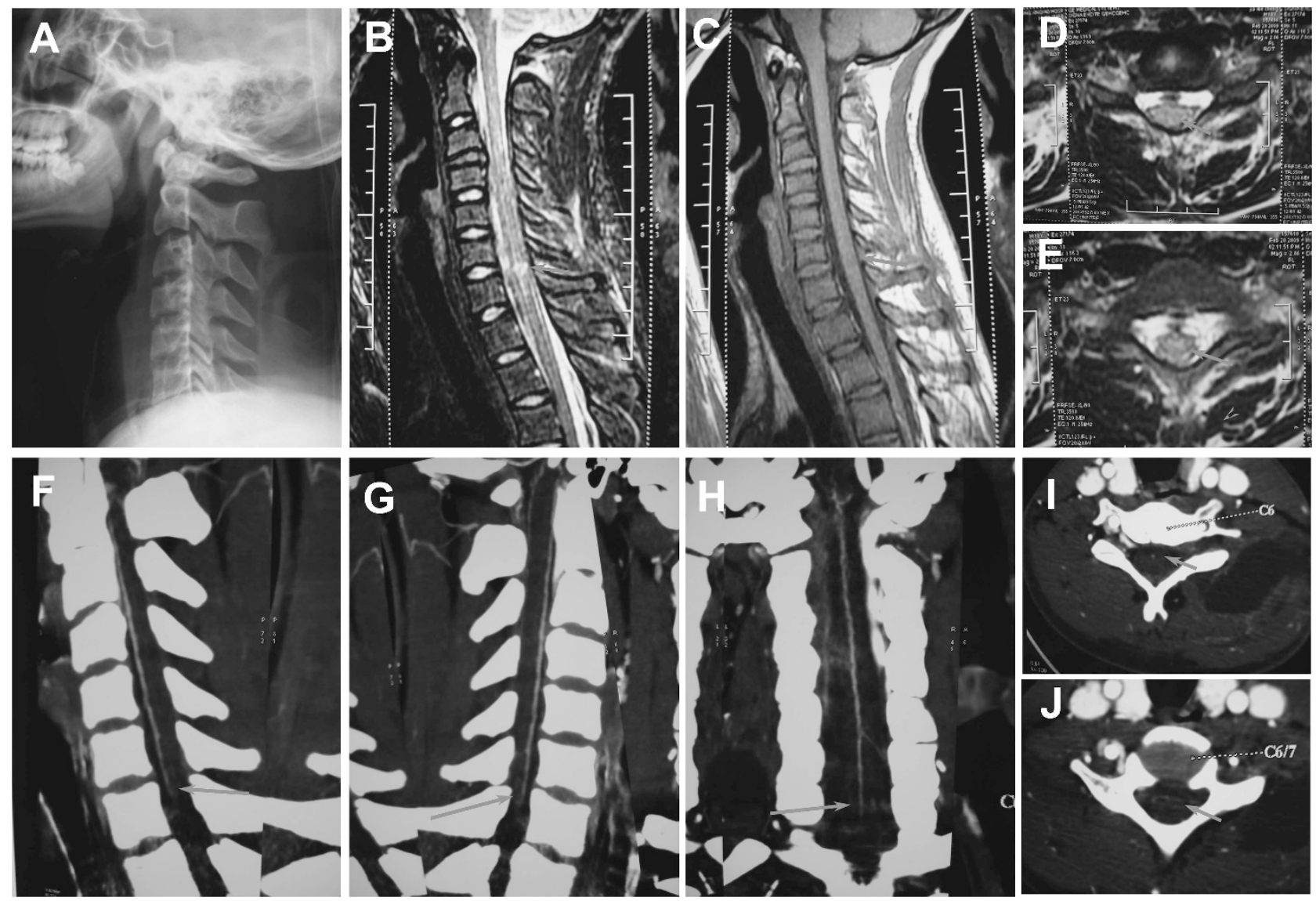

Figure 3 Patient 14. A 18-year-old man with a stab wound at C6/7. (A) Lateral radiograph showing no cervical fracture and dislocation. (B) Sagittal T2-weighted MRI showing cord hyperintensity at C6/7(arrow). (C) Sagittal T1-weighted MRI showing atypical intramedullary hypointensity (arrow). (D, E) Transverse section T2-weighted MRI showing cord hyperintensity at C6/7. (F-H) Sagittal and coronal views CTA showing ASA rupture and spinal cord relative hypoperfusion at C6/7. (I) Transverse section CTA showing no ASA rupture at C6. (J) Transverse section CTA showing ASA rupture at C6/7. A full color version of this figure is available at the Spinal Cord journal online.

anterior two thirds of the cord tissue by central and pial branches. Numerous anterior ARAs supply the spinal cord throughout its length by anastomoses, which branch upward and downward. The posterior spinal arteries are usually paired, course on the posterolateral surface of the spinal cord along its entire length, and may occasionally be discontinuous. ARAs and posterior spinal arteries are less than $0.5 \mathrm{~mm}$ in diameter, have no blood flow in either direction, ${ }^{8}$ and, at present, can only occasionally be depicted in vivo by catheter angiography. Thus, visualization of ASA is able to depict spinal cord blood supply in normal spinal cord and, to some extent, spinal cord pathological ischemia.

Catheter, CT and MRA are the ultimate imaging technique for diagnosing, localizing and classifying spinal vascular lesions. Despite its superior spatial resolution and image quality, catheter angiography has several major drawbacks, including being an invasive technique, ionizing radiation exposure, risk for major complications and can only be performed by experts. ${ }^{9,10}$ MRA benefits include having a strong background of suppression techniques, which allow the depiction of vessels smaller than the voxel size at acquisition. ${ }^{11}$ Moreover, reconstruction three-dimensional images of two-phase and time-resolved MRA is sufficient for the detection of the adamkiewicz artery and vessels of interest. ${ }^{12-14}$ However, MRA does not allow for visualization of the spinal cord and bone anatomy. This is why we chose CTA for visualizing ASA, although its disadvantages include inherent exposure to ionizing radiation and the requirement of administering a potentially nephrotoxic iodine contrast agent. The contrast injection parameters in this paper are based on the preliminary experiments and related literature. ${ }^{15,16}$

\section{ASA rupture in SCI}

In addition to spinal fracture and/or dislocation, SCI includes neural tissue and vascular damage at the site of contusion. However, to our knowledge, no studies have detected ASA rupture or injury in SCI patients. It is interesting that no ASA ruptures were found in 19 patients of our 20 SCI patients (even in six sever cervical fracture and dislocation ASIA A patients). This indicates that the spinal cord is more easily injured than the ASA in its anterior surface. Although ischemia is a key factor and key contributor to the secondary pathogenesis of SCI, ARAs injuries, including ruptured ARAs, occluded ARAs, or compressed ARAs (caused by the swelling of the surrounding tissue of the ARAs), are one possible cause of spinal cord ischemia but not ASA ruptures. For the ASA rupture of the stabwound patient, no neurological improvement was found, which indicates that individuals who experience ASA-rupture SCI have an even worse prognosis than individuals who experience ARA injuries. For visualization of ARAs in SCI, we failed with catheter, CT and 
MRA (data not show). Advancing imaging techniques will definitely further improve the possibility of non-invasively visualizing intradural spinal cord arteries and veins of SCI patients.

\section{Correlation between spinal cord ischemia and ASA rupture}

Acute traumatic SCI includes ischemia, axonal damage, swelling, edema, and hemorrhaging. All of these pathological changes demonstrate irregular T2 hyperintensity signals. The extent to which ischemia contributes to these SCI pathologies cannot be determined. However, spinal cord ischemia signal intensity is correlated with pathological changes, such as location and extent of the cord edema, which can be observed through MRI. Subtle spinal cord ischemia was found in BSS and CCS patients, while distinct spinal cord ischemia was found in tetraplegia patients. In our studies, no ASA ruptures were found in any BSS, CCS or tetraplegia patients (except one stabwound patient), which suggests that spinal cord ischemia is not associated with ASA rupture. Spinal cord ischemia maybe correlated with primary SCI and a reduction of ARAs blood supply.

Neurological recovery is, to some extent, related to the degree of ischemic neuronal damage for acute spinal cord ischemia syndrome. ${ }^{17}$ In animal models, the duration of the ischemia is related to histological damage and motor recovery. ${ }^{18}$ We agree with Iseli et al. ${ }^{19}$ that the severity of the initial neurological injury constitutes the main factor, which determines the prognosis in traumatic spinal cord injuries. Waters et al. ${ }^{20,21}$ pointed out that less than $1 \%$ of paraplegics reach ambulatory ability in the community if they do not present motor contraction in their lower limbs within a month after injury. If motor power develops within a month after injury, this percentage increases greatly for both paraplegia and incomplete tetraplegia $(47.0 \%, 42.1 \%$, respectively). In our studies, we followed up with all $20 \mathrm{SCI}$ and ischemia patients for over 6 months. The neurological deficit, classified according to the ASIA impairment scale, improved in the subtle spinal cord ischemia BSS and CCS patients (70\%). This is not only because of increased spinal cord blood supply but also because the original spinal cord injuries were not severe. Thirty percent of tetraplegia patients with distinct spinal cord ischemia have an unfavorable neurological recovery prognosis. Our results show that, aside from the initial neurological injury, ischemia determines the prognosis of neurological improvement, especially for incomplete tetraplegia patients.

\section{CONCLUSION}

Understanding the details of blood supply to the spinal cord after trauma is crucial for the ongoing discussion about the secondary pathogenesis of SCI, which influences the chronic functional deficits and necessity of early decompression. Although no ASA rupture was found, for blunt cervical fracture and dislocation patients, neurological prognosis after SCI has a close correlation with ischemia without ASA rupture. The limitations of this preliminary study include, visualization of ARAs, blinded CT perfusion measurements, limited patient population and CTA and MRI taken during follow-up.

\section{DATA ARCHIVING}

There were no data to deposit.

\section{CONFLICT OF INTEREST}

The authors declare no conflict of interest.

1 Young W. Secondary injury mechanisms in acute spinal cord injury. J Emerg Med 1993; 11(Suppl 1): 13-22.

2 Kuker W, Weller M, Klose U, Krapf H, Dichgans J, Nagele T. Diffusion-weighted mri of spinal cord infarction-high resolution imaging and time course of diffusion abnormality. J Neurol 2004; 251: 818-824.

3 Novy J, Carruzzo A, Maeder P, Bogousslavsky J. Spinal cord ischemia: clinical and imaging patterns, pathogenesis, and outcomes in 27 patients. Arch Neurol 2006; 63: 1113-1120

4 Backes WH, Nijenhuis RJ. Advances in spinal cord MR angiography. AJNR Am J Neuroradiol 2008; 29: 619-631.

5 DiTunno JF, Young W, Creasey G. International standards for neurological and functional classification of spinal cord injury: revised 1992. Paraplegia 1994; 32: 70-80.

6 Yagi M, Ninomiya K, Kihara M, Horiuchi Y. Long-term surgical outcome and risk factors in patients with cervical myelopathy and a change in signal intensity of intramedullary spinal cord on magnetic resonance imaging. J Neurosurg Spine 2010; 12: 59-65.

7 Thron A. Vascular Anatomy of the Spine. Oxford University Press: Oxford, 2002

8 Krauss WE. Vascular anatomy of the spinal cord. Neurosurg Clin N Am 1999; 10: 9-15.

9 Savader SJ, Williams GM, Trerotola SO, Perler BA, Wang MC, Venbrux AC et al. Preoperative spinal artery localization and its relationship to postoperative neurologic complications. Radiology 1993; 189: 165-171.

10 Kieffer E, Fukui S, Chiras J, Koskas F, Bahnini A, Cormier E. Spinal cord arteriography: a safe adjunct before descending thoracic or thoracoabdominal aortic aneurysmectomy. J Vasc Surg 2002; 35: 262-268.

11 Nijenhuis RJ, Leiner T, Cornips EM, Wilmink JT, Jacobs MJ, van Engelshoven JM et al. Spinal cord feeding arteries at MR angiography for thoracoscopic spinal surgery: feasibility study and implications for surgical approach. Radiology 2004; 233: 541-547.

12 Bley TA, Duffek CC, François CJ, Schiebler ML, Acher CW, Mell M et al. Presurgical localization of the artery of Adamkiewicz with time-resolved 3.0-T MR angiography. Radiology 2010; 255: 873-881.

13 Sheehy NP, Boyle GE, Meaney JF. Normal anterior spinal arteries within the cervical region: high-spatial-resolution contrast-enhanced three-dimensional MR angiography. Radiology 2005; 236: 637-641

14 Nijenhuis RJ, Mull M, Wilmink JT, Thron AK, Backes WH. MR angiography of the great anterior radiculomedullary artery (Adamkiewicz artery) validated by digital subtraction angiography. AJNR Am J Neuroradiol 2006; 27: 1565-1572.

15 Ou P, Schmit P, Layouss W, Sidi D, Bonnet D, Brunelle F. CT angiography of the artery of Adamkiewicz with 64-section technology: first experience in children. AJNR Am J Neuroradiol 2007; 28: 216-219.

16 Zhao SH, Logan L, Schraedley P, Rubin GD. Assessment of the anterior spinal artery and the artery of Adamkiewicz using multi-detector CT angiography. Chin Med J (Engl) 2009; 122: 145-149.

17 Little JW, Goldstein B, Gitter A, Haselkorn JK. Spinal cord infarction: varying degrees of upper and lower motoneuron dysfunction. J Spinal Cord Med 1996; 19: 242-248.

18 Gelfan S, Tarlov IM. Interneurons and rigidity of spinal cord origin. J Physiol (London) 1959; 146: 594-617.

19 Iseli E, Cavigelli A, Dietz V, Curt A. Prognosis and recovery in ischaemic and traumatic spinal cord injury: clinical and electrophysiological evaluation. J Neurol Neurosurg Psychiatry 1999; 67: 567-571.

20 Waters RI. Functional prognosis of spinal cord injuries. J Spinal Cord Med 1996; 19 : 89-92.

21 Waters RL, Adkins R, Yakura J, Sie I. Donal Munro Lecture: functional and neurologic recovery following acute spinal cord injury. J Spinal Cord Med 1998; 21: 195-199. 